\title{
POSSIBLE INSTABILITY OF THE VACUUM \\ IN A STRONG MAGNETIC FIELD
}

\author{
Giorgio CALUCCI* \\ Dipartimento di Fisica Teorica dell'Università di Trieste, Trieste, I 34014 Italy \\ INFN, Sezione di Trieste, Italy
}

\begin{abstract}
The possibility that a static magnetic field may decay through production of electron positron pairs is studied. The conclusion is that this decay cannot happen through production of single pairs, as in the electric case, but only through the production of a many-body state, since the mutual magnetic interactions of the created pairs play a relevant role.

The investigation is made in view of the proposed existence of huge magnetic field strengths around some kind of neutron stars.
\end{abstract}

\footnotetext{
* E-mail: giorgio@ts.infn.it
} 


\section{Introduction}

The perturbative calculations in QED have been pushed to an exceptional degree of refinement as far as their comparison with the high precision experiments. The study of the situations which require a genuine non-perturbative treatment is less systematic, also in this field, however, there are problems that can be considered classical and have gained a high degree of clarification. One of these problems concerns the effects of intense external field: the decay of the vacuum subject to a strong electric field through emission of $e^{-} e^{+}$pairs is well settled through the analysis given by Schwinger, starting from the Euler-Heisenberg effective Lagrangian[1,2]. If one considers strong magnetic fields there is a strict analogy if we admit the existence of monopoles, in that case the problem is only quantitative since the monopoles are expected to be very heavy, but for ordinary particles the parallel with the electric case is less straightforward.

The difference is that we associate a potential energy to the charge in an electric field, but we cannot do the same for an electric charge in a static magnetic field. The possible instability of the vacuum in the magnetic field, on the other side, seems interesting to be analyzed in view of the guess that extremely high magnetic field, of macroscopic extension, are realized in nature, around some neutron stars[3]. If the magnetic field is not completely static there is certainly a pair production, it can be seen $e . g$. treating the problem with the formalism of the adiabatic approximations[4], but in this case the rate of production depends on the square of the time derivative of the field. Here a qualitative and partially quantitative analysis of the possibility that the some kinds of vacuum instability leads to pair production even in static conditions is presented.

The main idea is stated in this way: any number of pairs in a given magnetic field cannot give rise to instabilities unless we take to some extent into account also their mutual interaction: in fact in this last case every pair shields partially the magnetic field in which the other particles lie, the overall effect could be a decreasing of the field intensity. This effect, when the original field is very strong, may decrease the density of magnetic energy and compensate the cost in energy for the creation of the pairs of charged particles. If this is true the effect is largely collective and cannot be studied particle by particle. A simple energy balance of this collective production is presented in section 2 and the reason for believing in the instability are given, since at this point we are in presence of an $e^{-} e^{+}$multiparticle state the superimposed Coulomb effects are studied in section 3, some conclusions are presented in the last section.

\section{Main features of the model}

The physical model is described in these terms: there is a classical magnetic field $\vec{B}$ constant in time and in it there is a second quantized electron field $\Psi$. The 
total energy of the system is

$$
\mathcal{E}_{T}=\frac{1}{2} \int B^{2} d^{3} r+\mathcal{H} \quad \text { with } \quad \mathcal{H}=\int\left\{\Psi^{\dagger}[\vec{\alpha} \cdot(\vec{p}+e \vec{A})+\beta m] \Psi\right\} d^{3} r
$$

The usual variational principle, for the stationary case, $\delta \mathcal{E}_{T}=0$ yields the equation for the magnetic field. This equation is then brought to numerical form by putting it among eigenstates of $\mathcal{H}$. Two known relations are used :

$$
<\sigma\left|\partial_{s} \mathcal{H}\right| \sigma>=\partial_{s}<\sigma|\mathcal{H}| \sigma>=\partial_{s} E_{\sigma}(s)
$$

when $\mathcal{H}$ depends on the parameter $s, \mid \sigma>$ is one of its eigenstates and $E_{\sigma}$ is the corresponding eigenvalue. Since $E$ will be expressed in terms of $\vec{B}=\operatorname{curl} \vec{A}$, one must remember that:

$$
\frac{\delta}{\delta A_{i}(\vec{r})}=\epsilon_{i k l} \partial_{k} \frac{\delta}{\delta B_{l}(\vec{r})}
$$

The standard relation $\operatorname{curl} \vec{B}=\vec{J}$, yields then

$$
\epsilon_{i k l} \partial_{k} B_{l}=-\epsilon_{i k l} \partial_{k} \frac{\delta}{\delta B_{l}} E \quad \text { i.e. } \quad B_{l}=-\frac{\delta}{\delta B_{l}} E+\partial_{l} U
$$

The functional derivative is zero in the limit $e \rightarrow 0$, so $\partial_{l} U$ represents the field in absence of vacuum polarization, it will be denoted by $B_{l}^{(o)} *$.

Now we specialize to a definite family of field configurations: let the magnetic field be uniform $\vec{B}=B \vec{n}$ and the volume of quantization of the system be a prism of height $L$, parallel to $\vec{n}$, and square section of side $R$. The multiparticle state is obtained in the most trivial way i.e. by filling the one-particle states until some Fermi level and leaving completely empty the higher one-particle states. It turns out natural to consider two independent parameters in order to fix the highest populated level one for the longitudinal and one for the transverse degrees of freedom; so it results (see e.g. [6]):

$$
\sum_{\text {levels }}=\frac{L}{2 \pi} \int_{-K}^{K} d k \cdot e B \frac{R^{2}}{2 \pi} \cdot \sum_{o}^{N} \sum_{s= \pm 1}
$$

Looking for a uniform solution $\vec{B}^{(o)}$ must be a constant vector and $-\delta E / \delta B_{l}$ is also a constant playing the role of a total magnetization $M_{l}$.

* A clear although artificial way of dealing with this kind of boundary condition may be found in the formalism of space-dependent coupling constants as proposed by Bogoliubov and Shirkov[5]. 
The energy coming from $\mathcal{H}$ has a constant density $\epsilon=E / V, V=R^{2} L$. For notational simplicity all the vectors are projected onto the direction $\vec{n}$ and only these components are now used. The total energy density is

$$
\epsilon_{T}=\frac{1}{2} B^{2}+\epsilon=\frac{1}{2}\left(B^{(o)}+M\right)^{2}+\epsilon,
$$

and if it can become smaller that the energy density $\frac{1}{2} B^{(o)^{2}}$, we may argue that the vacuum polarization leads to an instability of the magnetic vacuum.

The single-particle energy levels are the standard relativistic Landau levels

$$
w_{n, s}(k)=\sqrt{m^{2}+k^{2}+e B(2 n+1+s)}
$$

so the eigenvalue of $\mathcal{H}$ is

$$
E(K, N)=2 \cdot \frac{1}{4 \pi^{2}} L R^{2} e B \int_{-K}^{K} d k\left[2 \sum_{n=1}^{N} \sqrt{m^{2}+k^{2}+2 e B n}+\sqrt{m^{2}+k^{2}}\right] .
$$

The factor 2 in front of the whole expression arises when the contribution of the positron states is added to the contribution of the electron states. In order to perform the discrete sum the approximation used is

$$
\sum_{n=1}^{N} \sqrt{F+G n} \approx \frac{2}{3 G}\left[F+\left(N+\frac{1}{2}\right) G\right]^{3 / 2}
$$

which is valid up to terms constant in $N$.

In the actual situation $F=m^{2}+k^{2}, G=2 e B$. The integration over $k$ may be carried out completely in a straightforward way; the result is complicated and not very transparent. Since we are looking for a possible instability we are free to choose the trial state and so the range of the parameters $N$ and $K$, a choice that seems promising is

$$
N e B>>K^{2}>>e B \quad, \quad K>>m,
$$

because it allows an expansion in decreasing powers of $\sqrt{\mathrm{NeB}}$.

$$
\epsilon=\frac{1}{3 \pi^{2}}\left[2 K(2 N e B)^{3 / 2}+K^{3} \sqrt{2 N e B}\right]+\cdots=a B^{3 / 2}+c \sqrt{B}+d+\cdots .
$$

The coefficients $a, c$ are defined in the expression, $d$ denotes the addendum which does not contains the factor $\mathrm{NeB}$.

This expression is now used to calculate $M$ :

$$
M=-\frac{3}{2} a \sqrt{B}-\frac{1}{2} c / \sqrt{B}+\cdots .
$$


Then it is inserted into the expression for the total energy difference

$$
\Delta=\frac{1}{2} B^{2}+\epsilon-\frac{1}{2}\left[B^{(o)}\right]^{2}=\epsilon+M B-\frac{1}{2} M^{2},
$$

with the result:

$$
\Delta=-\frac{1}{2} a B^{3 / 2}-\frac{9}{8} a^{2} B+\frac{1}{2} c \sqrt{B}-\left(\frac{3}{4} a c-d\right) .
$$

The coefficient of the leading power in $B$ is negative and we may therefore conclude that there must exist configurations in which the creation of a collective state of electrons and positrons has the effect of lowering the total energy notwithstanding the cost in energy of the mass and kinetic terms for the charged particles.

\section{Effect of the Coulomb interaction}

Since we are now considering the creation of a plasma of $e^{+}, e^{-}$we are also led to consider the possible effect of the Coulomb interaction. It may be estimated perturbatively by inserting the corresponding two-body operator:

$$
\mathcal{V}_{c}=\frac{1}{2} \iint \Psi^{\dagger}(\vec{r}) \Psi(\vec{r}) \frac{\alpha}{|\vec{r}-\vec{s}|} \Psi^{\dagger}(\vec{s}) \Psi(\vec{s}) d^{3} r d^{3} s
$$

between the original states. This interaction is expected to give a negative contribution to the energy and since it does not contain $\vec{A}$ it does not modify the magnetization. The procedure to deal with this problem can be found in standard textbooks[7], the actual calculations become, however, very laborious if we want to use the correct wave functions of the electron, which are essentially harmonicoscillator functions. A simplified investigation is here presented: the particle states are simply represented by plane waves, the sums are cut at the same levels as in the previous case, so a maximum longitudinal momentum $K$ is used and a maximum transverse momentum $P$ is introduced, with the later identification $P^{2}=N e B$. In this way it is possible to get a simple estimate of the Coulomb effect, in considering the pair interaction we obtain the overall cancellation of the diagonal terms $\left(e^{-}, e^{-}\right),\left(e^{+}, e^{+}\right)$with $\left(e^{-}, e^{+}\right)$, which in this case is particularly evident, whereas the exchange terms survive and the contributions of the negative and positive charge add up; the result it obviously definite negative and may be expressed as:

$$
E_{c}=-4 \pi V \frac{1}{(2 \pi)^{6}} \iint \frac{\alpha}{\left(\vec{p}_{1}-\vec{p}_{2}\right)^{2}} d^{3} p_{1} d^{3} p_{2} \cdot 2
$$

the last factor 2 comes here also from the sum over the two charge states.

The integrand depends only on the difference of the momenta, so three integrations are easily performed; defining $\rho=K^{2} / P^{2}$ the result is

$$
I=\iint \frac{1}{\left(\vec{p}_{1}-\vec{p}_{2}\right)^{2}} d^{3} p_{1} d^{3} p_{2}=128 K^{2} P^{2} \int_{o}^{1} \frac{(1-u)(1-u)(1-w)}{u^{2}+v^{2}+\rho w^{2}} d u d v d w
$$


In the limit $\rho \rightarrow 0$ i.e. $P>>K$ the integral develops a logarithmic singularity which gives the leading term:

$$
I \approx 16 \pi K^{2} P^{2}\left[\ln P^{2} / K^{2}+\text { const }\right]
$$

With the identification $P^{2}=N e B$ the leading term of the Coulomb energy is:

$$
\epsilon_{c}=-\frac{\alpha}{8 \pi^{5}} I=-\frac{2 \alpha}{\pi^{4}} N e B K^{2} \ln \left(N e B / K^{2}\right)
$$

Comparing it with the results of eq. (6) we see that the Coulomb energy is definitively sub-leading. This results gives us confidence that higher order perturbative corrections, which may also affect the magnetization, will not destroy the main result of the previous chapter.

\section{Conclusions}

The main conclusion we may draw from this particularly simple model is that the indications of an instability of the magnetic vacuum are confirmed. The source of this instability is to be found in the dependence of the energy density of the electron field on the magnetic field. The leading term grows as $\epsilon_{o} \propto B^{\sigma}$ and is obviously positive, the diamagnetic term has a leading addendum $B\left(\partial \epsilon_{o} / \partial B\right)$, it carries a minus sign, and since $1<\sigma<2$ this term over-compensates the positive amount of energy required for the creation of pairs, the applied magnetic field $B^{(o)}$ is reduced by the pair creations, it results in fact, for the actual case $\sigma=\frac{3}{2}$ $B \approx B^{(o)}-\frac{3}{2} a \sqrt{B^{(o)}}+\frac{9}{8} a^{2}$; the effects of the Coulomb interaction are seen only in the sub-leading terms. The requirement of very large magnetic field is essential in order that the classification in leading and sub-leading terms be meaningful, in fact looking blindly at eq (8) one would get the impression that the total energy continuously decreases with increasing $N$, this is clearly impossible, for a given $B^{(o)}$ at a certain value of $N$, the resulting field $B$ becomes too small for the whole treatment to be correct. The determination of the actual values of $B^{(o)}$ for which the process of spontaneous pair creation can happen is not possible within the present treatment; the main problem in performing a quantitative estimate of the process lies in the fact that it proceeds necessarily via a tunnel effect, because the creation of a small number of pairs is not enough to lower the magnetic energy.

In fact the possible existence of the instability of the magnetic vacuum is strictly related to the mutual interaction of the created electron pairs, expressed by the fact that they are located in the field modified by the existence of the other pairs. The existence of an instability when only non interacting pairs are considered is excluded, in a static field, because under these conditions, the effective Euler-Heisenberg Lagrangian, which describes the effect of virtual charged spinors in a given external field, never develops an imaginary part, contrary to what happens for the electrostatic case $[1,8]$. The situation is also different from what expected in the spin-one 
case, where for very intense magnetic fields instabilities are expected already at the level of single pair creation[9], but the known charged particles with spin one are much heavier than the electron so there should not be sizable interference between the two processes at the foreseen field strengths[3] of the order of $10^{10} \mathrm{~T}$.

It must be, finally, noted the all the virtual effects have been ignored, they are likely to be important for very large field strengths, because they renormalize the electron charge and may also destroy the spherical symmetry of the Coulomb interaction[10], also the photon degrees of freedom coupled with the electron field may have a role, here only static interactions were analyzed, but all these and possibly other dynamical features are superimposed complications, they do not give, however, any indication of being able to destroy the main conclusion that emerges from the simple analysis presented in section 2 .

\section{Acknowledgments}

This work has been partially supported by the Italian Ministry of the University and of Scientific and Technological Research by means of the Fondi per la Ricerca scientifica - Università di Trieste.

\section{References}

1. J.Schwinger, Phys. Rev. 82, 664 (1951) and Phys. Rev. 93, 615 (1954).

2. V.I Ritus: The Lagrangian function of an intense electromagnetic field A.I.Nikishov: The S-matrix of QED with pair creating external field in Issues in intense field QED; ed. V.L. Ginzburg - Nova scientia pub. N.Y. 1987.

Qiong-gui Lin J.Phys. G25,17 (1999)

3. C.Thompson, R.C.Duncan, Astrophys. J. 408, 194 (1993);

H. Hanami, Astrophys. J. 491, 687 (1997);

K. Hurley et al. , Nature 397, 41 (1999);

M. Feroci et. al., Astrophys. J. 515, L9 (1999).

4. G. Calucci Modern Phys. L. A 14, 1183 (1999).

5. N.N. Bogoliubov, D.V.Shirkov: Introduction to the theory of quantized fields - Ch.VI Interscience Pub. New York 1959.

6. L.D.Landau, E.M.Lifshits: Quantum mechanics - Ch. XV $₫ 112$ Pergamon Press Oxford 1977.

7. L.D.Landau, E.M.Lifshits: Statistical Physics - Ch. VII -80 Pergamon Press Oxford 1980.

8. J.S.Schwinger Particles, Sources and Fields, vol.2, Ch.4 Addison Wesley 1973.

9. N.K.Nielsen and P.Olesen N.Phys. B144 (1978), 376;

J. Ambjorn and P.Olesen Nucl Phys B315 (1989), 606.

10. R. Ragazzon, J. Phys. A: Math. Gen 25, 2997 (1992);

G. Calucci, R.Ragazzon J. Phys. A: Math. Gen 27, 2161 (1994). 